\title{
Integrals of Jacobi Functions
}

\section{By Shyam L. Kalla, Salvador Conde and Yudell L. Luke}

\begin{abstract}
In this paper, we study $\int_{-1}^{1}(1-x)^{a}(1+x)^{b} P_{v}^{(\alpha, \beta)}(x) d x$ and its partial derivatives with respect to $a$ and $b$, where $P_{v}^{(\alpha, \beta)}(x)$ is the Jacobi function. Our expressions generalize the work of Blue, Gautschi and Gatteschi. The results are useful to derive integration formulas for integrands with algebraic and logarithmic singularities.
\end{abstract}

1. Introduction. In a previous paper Blue [2] gave a simple expression for $\int_{0}^{1}(\ln x) R_{n}(x) d x$, where $R_{n}(x)=P_{n}(2 x-1)$ is the shifted Legendre polynomial. In a follow-up note on this paper, Gautschi [4] treated

$$
\int_{-1}^{1}\{\ln (1+t)\}(1+t)^{b} P_{\nu}(t) d t
$$

where $P_{\nu}(t)$ is the Legendre function which reduces to the Legendre polynomial if $\nu$ is a positive integer. More recently Gatteschi [3] studied

$$
\int_{0}^{1} x^{\rho}(1-x)^{\alpha}(\ln x) R_{n}^{(\alpha, \beta)}(x) d x,
$$

where $R_{n}^{(\alpha, \beta)}(x)=P_{n}^{(\alpha, \beta)}(2 x-1)$ is the shifted Jacobi polynomial. The purpose of this paper is to generalize the above results by examining the integral

$$
I_{\nu, \alpha, \beta}^{a, b}=\int_{-1}^{1}(1-x)^{a}(1+x)^{b} P_{\nu}^{(\alpha, \beta)}(x) d x, \quad R(a)>-1, R(b)>-1,
$$

and its partial derivatives with respect to $a$ and $b$, where

$$
P_{\nu}^{(\alpha, \beta)}(x)=\frac{(\alpha+1)_{\nu}}{\Gamma(\nu+1)}{ }_{2} F_{1}\left(\begin{array}{c}
-\nu, \nu+\lambda \\
\alpha+1
\end{array} \mid \frac{1-x}{2}\right), \quad \lambda=\alpha+\beta+1,
$$

is the Jacobi function. Here and throughout this paper, we follow the notation of Luke [5], [6] and assume the parameters are such that the expressions make sense. Note that the ${ }_{2} F_{1}$ in (2) is unchanged if $\nu$ is replaced by $-\nu-\lambda$. It is convenient to introduce

$$
L_{\nu, \alpha, \beta}^{a, b}=\frac{(\beta+1)_{\nu}}{\Gamma(\nu+1)} \int_{-1}^{1}(1-x)^{a}(1+x)^{b}{ }_{2} F_{1}\left(\begin{array}{c}
-\nu, \nu+\lambda \\
\beta+1
\end{array} \mid \frac{1+x}{2}\right) d x=I_{\nu, \beta, \alpha}^{b, a},
$$

valid under the same conditions as for (1).

If $\nu$ is a positive integer or zero, call it $n$, then it follows from Luke [5, Volume 1 , p. 70, Eq. (4)] that

$$
P_{n}^{(\alpha, \beta)}(x)=\frac{(-)^{n}(\beta+1)_{n}}{n !}{ }_{2} F_{1}\left(\begin{array}{c}
-n, n+\lambda \\
\beta+1
\end{array} \mid \frac{1+x}{2}\right),
$$

Received November 21, 1980; revised March 9, 1981.

1980 Mathematics Subject Classification. Primary 33A65, Secondary 65D30. 
and this together with (2) gives

$$
P_{n}^{(\alpha, \beta)}(x)=(-)^{n} P_{n}^{(\beta, \alpha)}(-x) .
$$

Consequently

$$
I_{n, \alpha, \beta}^{a, b}=(-)^{n} I_{n, \beta, \alpha}^{b, a}=(-)^{n} L_{n, \alpha, \beta}^{a, b} .
$$

We can call (1) a beta integral of the Jacobi function. The beta integral of the Legendre function suitably modified by a constant multiplier is also included in (1) since the Legendre function on the cut is defined by

$$
\begin{aligned}
P_{\nu}^{\mu}(x)= & {[\Gamma(1-\mu)]^{-1}[(1+x) /(1-x)]^{\mu / 2} } \\
& \times{ }_{2} F_{1}\left(\begin{array}{c}
-\nu, \nu+1 \\
1-\mu
\end{array} \mid \frac{1-x}{2}\right), \quad-1<x<1 .
\end{aligned}
$$

By virtue of a Kummer transformation formula, we can write

$$
P_{\nu}^{(\alpha, \beta)}(x)=\frac{(\alpha+1)_{\nu}}{\Gamma(\nu+1)}\left(\frac{1+x}{2}\right)^{-\beta}{ }_{2} F_{1}\left(\begin{array}{c}
\alpha+1+\nu,-\nu-\beta \\
\alpha+1
\end{array} \mid \frac{1-x}{2}\right) .
$$

Similarly,

(9) $P_{\nu}^{\mu}(x)=[\Gamma(1-\mu)]^{-1} 2^{\mu}\left(1-x^{2}\right)^{-\mu / 2}{ }_{2} F_{1}\left(\begin{array}{c|c}1+\nu-\mu,-\mu-\nu & 1-x \\ 1-\mu\end{array}\right)$.

In what follows, we deal principally with (1). We have need for the beta integral

$$
\begin{aligned}
\int_{-1}^{1}(1-t)^{\rho}(1+t)^{\eta} d t=2^{\eta+\rho+1} \int_{0}^{1} t^{\eta}(1-t)^{\rho} d t \\
=\frac{2^{\eta+\rho+1} \Gamma(\eta+1) \Gamma(\rho+1)}{\Gamma(\eta+\rho+2)}, \quad R(\rho)>-1, R(\eta)>-1 .
\end{aligned}
$$

2. $I_{\nu, \alpha, \beta}^{a, b}$ and Related Integrals. From (1) and (2), using termwise integration and (10), we get

$$
\begin{array}{r}
I_{\nu, \alpha, \beta}^{a, b}=\frac{2^{a+b+1} \Gamma(a+1) \Gamma(b+1)(\alpha+1)_{\nu}}{\Gamma(\nu+1) \Gamma(a+b+2)}{ }_{3} F_{2}\left(\begin{array}{c}
-\nu, \nu+\lambda, a+1 \\
\alpha+1, a+b+2
\end{array} \mid 1\right) \\
R(a)>-1, R(b)>-1 .
\end{array}
$$

If the above ${ }_{3} F_{2}$ does not terminate, then, to insure absolute convergence, we must require $R(b+1-\beta)>0$. Again the above ${ }_{3} F_{2}$ is unchanged if $\nu$ is replaced by $-\nu-\lambda$. By a partial differentiation of (1) and (11) with respect to $a$, we have

$$
\begin{aligned}
J_{\nu, \alpha, \beta}^{a, b}= & \frac{\partial I_{\nu, \alpha, \beta}^{a, b}}{\partial a}=\int_{-1}^{1}\{\ln (1-x)\}(1-x)^{a}(1+x)^{b} P_{\nu}^{(\alpha, \beta)}(x) d x \\
= & {[\ln 2+\psi(a+1)-\psi(a+b+2)] I_{\nu, \alpha, \beta}^{a, b} } \\
& +\frac{2^{a+b+1} \Gamma(a+1) \Gamma(b+1)(\alpha+1)_{\nu}}{\Gamma(\nu+1) \Gamma(a+b+2)} \sum_{k=1}^{\infty} \frac{(-\nu)_{k}(\nu+\lambda)_{k}(a+1)_{k}}{(\alpha+1)_{k}(a+b+2)_{k} k !} \\
& \times\{\psi(a+1+k)-\psi(a+1)-\psi(a+b+2+k)+\psi(a+b+2)\} .
\end{aligned}
$$


Also

$$
\begin{aligned}
K_{\nu, \alpha, \beta}^{a, b} & =\frac{\partial I_{\nu, \alpha, \beta}^{a, b}}{\partial b}=\int_{-1}^{1}\{\ln (1+x)\}(1-x)^{a}(1+x)^{b} P_{\nu}^{(\alpha, \beta)}(x) d x \\
& =\frac{\partial L_{\nu, \beta, \alpha}^{b, a}}{\partial b}
\end{aligned}
$$

so that if $\nu$ is $n$,

$$
K_{n, \alpha, \beta}^{a, b}=\frac{\partial I_{n, \alpha, \beta}^{a, b}}{\partial b}=\frac{(-)^{n} \partial I_{n, \beta, \alpha}^{b, a}}{\partial b}=(-)^{n} J_{n, \beta, \alpha}^{b, a}
$$

It follows that

$$
\begin{gathered}
S_{\nu, \alpha, \beta}^{a, b}=\int_{-1}^{1}\left\{\ln \left(1-x^{2}\right)\right\}(1-x)^{a}(1+x)^{b} P_{\nu}^{(\alpha, \beta)}(x) d x \\
=J_{\nu, \alpha, \beta}^{a, b}+K_{\nu, \alpha, \beta}^{a, b}, \\
T_{\nu, \alpha, \beta}^{a, b}=\int_{-1}^{1}\{\ln (1-x) /(1+x)\}(1-x)^{a}(1+x)^{b} P_{\nu}^{(\alpha, \beta)}(x) d x \\
=J_{\nu, \alpha, \beta}^{a, b}-K_{\nu, \alpha, \beta}^{a, b} .
\end{gathered}
$$

Hence, if $a=b$ and $\alpha=\beta$,

$$
\begin{aligned}
S_{n, \alpha, \alpha}^{a, a} & =0 & & \text { if } n \text { is odd, } \\
& =2 J_{n, \alpha, \alpha}^{a, a} & & \text { if } n \text { is even, } \\
T_{n, \alpha, \alpha}^{a, a} & =0 & & \text { if } n \text { is even, } \\
& =2 J_{n, \alpha, \alpha}^{a, a} & & \text { if } n \text { is odd. }
\end{aligned}
$$

Replace $x$ by $2 x-1$ in the integral in (12). Then

$$
\begin{gathered}
J_{\nu, \alpha, \beta}^{a, b}=(\ln 2) I_{\nu, \alpha, \beta}^{a, b}+W_{\nu, \alpha, \beta}^{a, b}, \\
W_{\nu, \alpha, \beta}^{a, b}=2^{a+b+1} \int_{0}^{1}\{\ln (1-x)\}(1-x)^{a} x^{b} R_{\nu}^{(\alpha, \beta)}(x) d x,
\end{gathered}
$$

where $R_{\nu}^{(\alpha, \beta)}(x)=P_{\nu}^{(\alpha, \beta)}(2 x-1)$ is the shifted Jacobi function. Notice that

$$
W_{n, \alpha, \beta}^{a, b}=(-)^{n} W_{n, \beta, \alpha}^{b, a}+T_{n, \alpha, \beta}^{a, b}
$$

Thus evaluation of (20) follows from that of $I_{\nu, \alpha, \beta}^{a, b}$ and its partial derivative with respect to $a$.

An alternative representation for (1) follows from (8) and (10) by termwise integration. Thus

$$
\begin{aligned}
I_{\nu, \alpha, \beta}^{a, b}= & \frac{2^{a+b+1}(\alpha+1)_{\nu} \Gamma(b+1-\beta) \Gamma(a+1)}{\Gamma(\nu+1) \Gamma(a+b+2-\beta)} \\
& \times{ }_{3} F_{2}\left(\begin{array}{c}
-\nu-\beta, \nu+\alpha+1, a+1 \\
\alpha+1, a+b+2-\beta
\end{array} \mid 1\right),
\end{aligned}
$$

which is advantageous if $\nu+\beta$ is a positive integer or zero.

Since the Jacobi polynomial can also be written as

$$
P_{n}^{(\alpha, \beta)}(x)=2^{-n} \sum_{k=0}^{n}\left(\begin{array}{c}
n+\alpha \\
k
\end{array}\right)\left(\begin{array}{c}
n+\beta \\
n-k
\end{array}\right)(x-1)^{n-k}(x+1)^{k}
$$


we have the expansions

$$
\begin{gathered}
I_{n, \boldsymbol{\alpha}, \boldsymbol{\beta}}^{a, b}=\frac{2^{a+b+1}(-)^{n} \Gamma(b+1)(\beta+1)_{n} \Gamma(n+a+1)}{n ! \Gamma(n+a+b+2)} \\
\times{ }_{3} F_{2}\left(\begin{array}{c}
-n,-n-\alpha, b+1 \\
-n-a, \beta+1
\end{array}\right), \\
J_{n, \alpha, \beta}^{a, b}=[\ln 2+\psi(n+a+1)-\psi(n+a+b+2)] I_{n, \alpha, \beta}^{a, b} \\
+\frac{2^{a+b+1}(-)^{n} \Gamma(b+1)(\beta+1)_{n} \Gamma(n+a+1)}{n ! \Gamma(n+a+b+2)} \\
\times \sum_{k=0}^{n} \frac{(-n)_{k}(-n-a)_{k}(b+1)_{k}}{(-n-a)_{k}(\beta+1)_{k}}\{\psi(-n-a+k)-\psi(-n-a)\} .
\end{gathered}
$$

We have given two formulas for $I_{\nu, \alpha, \beta}^{a, b}$ for $\nu$ arbitrary and a third when $\nu$ is a positive integer $n$. Actually there are other repesentations which are a consequence of known relations between ${ }_{3} F_{2}$ 's of a unit argument. For a thorough treatment of this subject, see the volumes by Bailey [1], Luke [5], and Slater [7]. Equations (11) and (22) follow from

$$
\begin{array}{r}
{ }_{3} F_{2}\left(\begin{array}{c}
u, v, w \\
x, y
\end{array} \mid 1\right)=\frac{\Gamma(x) \Gamma(s)}{\Gamma(x-u) \Gamma(s+u)}{ }_{3} F_{2}\left(\begin{array}{c}
u, y-v, y-w \\
y, s+u
\end{array} \mid 1\right), \\
s=x+y-u-v-w .
\end{array}
$$

The series on the left is absolutely convergent if $R(s)>0$, while the same is true for that on the right if $R(x-u)>0$. Since the ${ }_{3} F_{2}$ on the right does not possess all the symmetry properties of that on the left, there are six different equations. In the case of (11), we need record only four of these since the ${ }_{3} F_{2}$ is invariant when $\nu$ is replaced by $-\nu-\lambda$. Let

$$
U={ }_{3} F_{2}\left(\begin{array}{c}
-\nu, \nu+\lambda, a+1 \\
\alpha+1, a+b+2
\end{array} \mid 1\right), \quad R(b+1-\beta)>0
$$

Then

$$
U=\frac{\Gamma(\alpha+1) \Gamma(b+1-\beta)}{\Gamma(\alpha-a) \Gamma(a+b+2-\beta)}
$$

$$
U=\frac{(a+b+2-\beta)_{\beta}}{(b+1-\beta)_{\beta}}{ }_{3} F_{2}\left(\begin{array}{c}
-\nu-\beta, \nu+\alpha+1, a+1 \\
\alpha+1, a+b+2-\beta
\end{array} \mid 1\right)
$$

$$
R(b)>-1,
$$

$$
\begin{array}{r}
U=\frac{(-\nu+b+1-\beta)_{\nu}}{(a+b+2)_{\nu}}{ }_{3} F_{2}\left(\begin{array}{r}
-\nu,-\nu-\beta, \alpha-a \\
\alpha+1,-\nu+b+1-\beta
\end{array} \mid 1\right), \\
R(\nu+a+b+2)>0,
\end{array}
$$

$$
\begin{gathered}
\times{ }_{3} F_{2}\left(\begin{array}{c}
-\nu-\lambda+a+b+2, \nu+a+b+2, a+1 \\
a+b+2-\beta, a+b+2
\end{array} \mid 1\right), \quad R(\alpha-a)>0, \\
U=\frac{\Gamma(\alpha+1) \Gamma(b+1-\beta)}{\Gamma(-\nu-\beta) \Gamma(\nu+\alpha+b+2)}{ }_{3} F_{2}\left(\begin{array}{c}
\nu+\lambda, \nu+a+b+2, b+1 \\
\nu+\alpha+b+2, a+b+2
\end{array} \mid 1\right),
\end{gathered}
$$$$
R(\nu+\beta)>0 \text {. }
$$ 
Another general formula is

$$
\begin{array}{r}
{ }_{3} F_{2}\left(\begin{array}{c}
a, b, c \\
e, f
\end{array} \mid 1\right)=\frac{\Gamma(e) \Gamma(f) \Gamma(s)}{\Gamma(a) \Gamma(s+b) \Gamma(s+c)}{ }_{3} F_{2}\left(\begin{array}{c}
e-a, f-a, s \\
s+b, s+c
\end{array} \mid\right), \\
s=e+f-a-b-c .
\end{array}
$$

Using (27) and (32) with $a$ replaced by $a+1, b=-\nu, c=\nu+\lambda, e=\alpha+1$, and $f=a+b+2$, and the reflection formula for gamma functions, we get

$$
\begin{aligned}
U= & \frac{\Gamma(\alpha+1) \Gamma(a+b+2) \Gamma(\nu+\beta-b) \sin \pi(\nu+\beta-b)}{\Gamma(a+1) \Gamma(\beta-b) \Gamma(\nu+\alpha+b+2) \sin \pi(\beta-b)} \\
& \times{ }_{3} F_{2}\left(\begin{array}{c}
\alpha-a, b+1, b+1-\beta \\
-\nu+b+1-\beta, \nu+\alpha+b+2
\end{array} \mid \begin{array}{l}
1
\end{array}\right), \quad R(a+1)>0 .
\end{aligned}
$$

In view of symmetry, two other forms can be deduced from (32). They do not appear to be interesting and so are omitted. The above forms are valuable because, for special values of the parameters, one of the ${ }_{3} F_{2}$ 's might terminate or might be easily summed in terms of gamma functions. In illustration, $U$ as given by (27) terminates if $\nu$ is a positive integer; likewise for (28) if $\nu+\beta$ is a positive integer. Thus, if none of the numerator parameters in (28) is a negative integer, but $\nu+\beta$ is a negative integer, then (28) is preferred over (27). Again for special values of the parameters, one of the ${ }_{3} F_{2}$ 's might reduce to a ${ }_{2} F_{1}$ which can always be summed. In this connection both (11) and (22) simplify if $a=\alpha$. The ${ }_{3} F_{2}$ on the left of (26) is said to be Saalschützian if $s=1$. If, in addition, the ${ }_{3} F_{2}$ terminates, then it can be summed.

Another representation for $I_{\nu, \alpha, \beta}$ follows from a formula which expresses a ${ }_{3} F_{2}$ of unit argument in terms of two other ${ }_{3} F_{2}$ 's of unit argument; see [5, Volume 1, p. 104, Eq. (11)]. Application of this result when $b$ and $\nu$ are positive integers has been discussed by Luke [5, Volume 1, pp. 281-283]. We have made no attempt to give all possible representations for $I_{\nu, \alpha, \beta}^{a, b}$ which ensue from transforms of ${ }_{3} F_{2}$ 's of unit argument. To get other formulas, see the discussion given in [5, Volume 1, pp. 103-109].

3. Special Cases. In this section, we illustrate simplified forms which emerge from the formulas in the last section. Let $\alpha-a=-m, m$ a positive integer or zero. Then, from (11) and (29), we get with the aid of the reflection formula for gamma functions

$$
\begin{aligned}
I_{\nu, \alpha, \beta}^{\alpha+m, b}= & \frac{2^{m+\alpha+b+1} \Gamma(b+1) \Gamma(m+\alpha+1) \Gamma(\nu+\alpha+1) \Gamma(\nu+\beta-b) \sin \pi(\nu+\beta-b)}{\Gamma(\alpha+1) \Gamma(\beta-b) \Gamma(\nu+1) \Gamma(\nu+m+\alpha+b+2) \sin \pi(\beta-b)} \\
& \times{ }_{3} F_{2}\left(\begin{array}{c}
-\nu,-m,-\nu-\beta \\
-\nu+b+1-\beta, \alpha+1
\end{array} \mid 1\right) .
\end{aligned}
$$

If $m=0$, this becomes

$$
I_{\nu, \alpha, \beta}^{\alpha, b}=\frac{2^{\alpha+b+1} \Gamma(b+1) \Gamma(\nu+\alpha+1) \Gamma(\nu+\beta-b) \sin \pi(\nu+\beta-b)}{\Gamma(\beta-b) \Gamma(\nu+1) \Gamma(\nu+\alpha+b+2) \sin \pi(\beta-b)},
$$

a result which follows directly from (11) or (22) since the ${ }_{3} F_{2}$ 's in each of these formulas becomes a ${ }_{2} F_{1}$ of unit argument. The same result also follows from (31). With $\nu=n$, (35) reduces to the form given by Gatteschi [3]. If $\alpha=\beta=0$ in (35), 
we get the result given by Gautschi [4]. We also have

$$
I_{\nu, \alpha, \beta}^{\alpha, \beta}=\frac{2^{\lambda} \Gamma(\beta+1) \Gamma(\nu+\alpha+1) \sin \pi \nu}{\pi \nu \Gamma(\nu+\lambda+1)},
$$

which vanishes if $\nu$ is an integer, zero excluded.

If $\nu$ is a positive integer $n$, the ${ }_{3} F_{2}$ in (11) terminates. If further $b=\beta$, it is Saalschützian and so can be summed. We find

$$
I_{n, \alpha, \beta}^{a, \beta}=\frac{2^{\beta+a+1} \Gamma(a+1) \Gamma(n+\beta+1) \Gamma(n+\alpha-a)}{n ! \Gamma(n+\beta+a+2) \Gamma(\alpha-a)} .
$$

When $\nu$ is a positive integer $n$, two other special cases follow upon application of results given by Luke [6, p. 165, Eqs. (12) and (13)]. (There in Eq. (12), the numerator of the right-hand side should be multiplied by $n !$.) These equations are omitted.

4. Computation by Use of Recursion Formulas. In [5, Volume 2, p. 147, Eqs. (64)-(66)], it is shown that

$$
f_{\nu}={ }_{3} F_{2}\left(\begin{array}{c|c}
-\nu, \nu+\lambda, a+1 \\
\alpha+1, a+b+2
\end{array} \mid 1\right)
$$

satisfies a third order difference equation in $\nu$ of the form

$$
f_{\nu}+\sum_{j=1}^{3} E_{j} f_{\nu-j}=0 .
$$

In the cited reference, we must put $z=1$ and correlate the notation. There $n$ is used in place of $\nu$. However, the results hold for $n$ arbitrary. We suppose, of course, the parameters are such that all expressions make sense. In theory, if we have a set of functions $f_{v+r}$ which satisfy (39) for $r=0,1,2$, we can use the recursion relation to evaluate $f_{\nu+m}, m=3,4, \ldots$ or $m=-1,-2, \ldots$ However, in practice difficulties might arise due to growth of round-off error. This subject has been rather thoroughly discussed in the literature and we shall not present complete details as it applies to the problem at hand. In this connection, see Luke [5], [6] and the references given there. In general, use of the recursion formula in the forward (backward) direction is stable if applied to the dominant (subdominant) solution. If the solution is neither dominant nor subdominant neither technique is stable and certain modifications must be made. For the case at hand, the three solutions of (39) all have algebraic behavior for $\nu$ large. Indeed for $\nu$ a large positive integer $\rightarrow+\infty$ there are three solutions proportional to $g_{1}=1, g_{2}=\cos \nu \pi$ and $g_{3}=$ $\nu \cos \nu \pi$. We have not determined which corresponds to $f_{\nu}$. However, no member of the set of solutions is decidedly dominant or subdominant to the others in that for $\nu$ large, $\left|g_{2} / g_{1}\right| \sim 1,\left|g_{2} / g_{3}\right| \sim 1 / \nu$. Consequently satisfactory results can be achieved by use of the recursion formula in the forward direction for moderately large $\nu$ provided normal round-off error controls are used.

Partial derivatives of $f_{\nu}$ with respect to any of the parameters can also be evaluated by recursion. From (39), for example,

$$
\begin{gathered}
g_{\nu}+\sum_{j=1}^{3} E_{j} g_{\nu-j}+\sum_{j=1}^{3} F_{j} f_{\nu-j}=0 \\
g_{\nu}=\frac{\partial f_{\nu}}{\partial a}, \quad F_{j}=\frac{\partial E_{j}}{\partial a} .
\end{gathered}
$$


We now outline an alternative scheme to compute $f_{\nu}$ by recursion. It is convenient to generalize and consider

$$
G_{m}=\frac{m !}{\left(\nu_{1}\right)_{m}}{ }_{3} F_{2}\left(\begin{array}{c}
\mu_{1}, \mu_{2}, \mu_{3} \\
m+\nu_{1}, \nu_{2}
\end{array} \mid z\right)
$$

We will derive a three-term recursion formula for $G_{m}$. For a sufficiently large integer $K$ evaluate $G_{K+1}$ and $G_{K}$ by means of the series (41). Then use the recursion relation in the backward direction to get $G_{K-1}, G_{K-2}, \ldots, G_{0}$. By proper identification of the parameters, $G_{0}=f_{v}$. To get the recursion formula use [5, Volume 2, pp. 135-137, Eqs. (8), (12)-(16)] with

$$
\begin{aligned}
j=1, s=3, r=2, t=4, z \text { replaced by } z / \lambda \text { and } \lambda \rightarrow \infty \\
\mu_{i}=1+a_{1}-b_{i}, i=1,2,3 ; \nu_{1}=1+a_{1}, \nu_{2}=1+a_{1}-a_{2} .
\end{aligned}
$$

Then in that notation $A_{4} \rightarrow 0$ and

$$
\begin{aligned}
G_{m}+\left(A_{1}+z B_{1}\right) G_{m-1}+\left(A_{2}+z B_{2}\right) G_{m-2}+\left(A_{3}+z B_{3}\right) G_{m-3}=0, & \\
A_{1}= & \frac{m}{\left(m-1+b_{s}\right)}\left[\left(m-2+b_{s}\right)-\left(m-1+b_{s}\right)\right], \\
B_{1} & =\frac{m\left(m-1+a_{r}\right)}{\left(m-1+b_{s}\right)}, \\
A_{2} & =\frac{m(m-1)}{2\left(m-1+b_{s}\right)}\left[\left(m-3+b_{s}\right)-2\left(m-2+b_{s}\right)+\left(m-1+b_{s}\right)\right], \\
B_{2} & =-\frac{m(m-1)}{\left(m-1+b_{s}\right)}\left(2 m+a_{1}+a_{2}+3\right), \\
B_{3} & =-A_{3}=\frac{m(m-1)(m-2)}{\left(m-1+b_{s}\right)} .
\end{aligned}
$$

Here $\left(m+p+b_{s}\right)$ stands for the product

$$
\left(m+p+b_{1}\right)\left(m+p+b_{2}\right)\left(m+p+b_{3}\right)
$$

and $\left(m+p+a_{r}\right)$ stands for $\left(m+p+a_{1}\right)\left(m+p+a_{2}\right)$, where $p$ is arbitrary. This is a four-term recursion formula. If $z=1$, the coefficient of $G_{m-3}$ is nil and we get the three-term expression

$$
G_{m}+W_{1} G_{m-1}+W_{2} G_{m-2}=0, \quad W_{i}=A_{i}+B_{i}, \quad i=1,2 .
$$

For the application at hand,

$$
\begin{gathered}
a_{1}=a+b+1, \quad a_{2}=a+b+1-\alpha, \\
b_{1}=a+b+2+\nu, \quad b_{2}=a+b+2-\nu-\lambda, \quad b_{3}=b+1,
\end{gathered}
$$

where $\lambda$ is now a new parameter. We are indebted to Professor Jet Wimp for the ideas of (41)-(44). He has also proved the following useful result with $z=1$. Let $R_{1}, R_{2}$ be the smallest positive integers such that

$$
-R_{1}<\mu_{i}, \quad \nu_{j} \leqslant R_{2}, \quad i=1,2,3, \quad j=1,2,
$$

and set

$$
P=2 R_{1}+3 R_{2}
$$


Let $M$ be an integer $(M>1)$ such that

$$
\begin{aligned}
& \frac{K M^{1 / 2}}{M-1}(M)_{\mu_{1}+P}\left(\frac{4}{27}\right)^{M}<\varepsilon \\
& \cdot K=\frac{2(3 \pi)^{1 / 2}\left|\Gamma\left(\nu_{2}\right)\right|}{\left|\Gamma\left(\mu_{1}\right) \Gamma\left(\mu_{2}\right) \Gamma\left(\mu_{3}\right)\right|}
\end{aligned}
$$

where $\varepsilon$ is the desired accuracy. Since $M$ will usually be rather large, we can replace (48) by

$$
K M^{\mu_{1}+P-1 / 2}(4 / 27)^{M}<\varepsilon
$$

Compute

$$
G_{M+P-j}^{*}=\sum_{k=0}^{2 M+R_{1}+2 j} \frac{\left(\mu_{1}\right)_{k}\left(\mu_{2}\right)_{k}\left(\mu_{3}\right)_{k}}{\left(\nu_{1}+M+P+j\right)_{k}\left(\nu_{2}\right)_{k} k !}, \quad j=0,1,
$$

and $G_{K}^{*}$ from

$$
G_{K}^{*}=-W_{1} G_{K-1}^{*}-W_{2} G_{K-2}^{*}, \quad K=M+P-1, M+P-2, \ldots, 0 .
$$

Then

$$
G_{0}^{*}=G_{0}+\varepsilon O\left(M^{\eta}\right), \quad M \rightarrow \infty, \quad \eta=\max \left(1,2-\nu_{2}\right) .
$$

The above ideas can be applied to get partial derivatives as in the discussion surrounding (38)-(40) We omit the details.

Acknowledgement. The present work is supported in part by CONDES of the Universidad del Zulia, through the research grant No. C716-79.

División de Postgrado

Facultad de Ingeniería

Universidad del Zulia

Maracaibo, Venezuela

División de Postgrado

Facultad de Ingeniería

Universidad del Zulia

Maracaibo, Venezuela

Department of Mathematics

University of Missouri

Kansas City, Missouri 64110

1. W. N. Bailey, Generalized Hypergeometric Series, Cambridge Univ. Press, Cambridge, 1935.

2. J. L. BLUE, "A Legendre polynomial integral," Math. Comp., v. 33, 1979, pp. 739-741.

3. L. Gatresch, "On some orthogonal polynomial integrals," Math. Comp., v. 35, 1980, pp. 1291-1298.

4. W. GAutschi, "On the preceding paper 'A Legendre polynomial integral' by James L. Blue," Math. Comp., v. 33, 1979, pp. 742-743.

5. Y. L. LuKE, The Special Functions and Their Approximations, Vols. 1, 2, Academic Press, New York, 1969.

6. Y. L. LUKE, Mathematical Functions and Their Approximations, Academic Press, New York, 1975.

7. L. J. Slater, Generalized Hypergeometric Functions, Cambridge Univ. Press, Cambridge, 1966. 